\title{
Magnetotransport properties of $\mathrm{Fe}_{0.8} \mathrm{Ga}_{0.2}$ films with stripe domains
}

\author{
M. Granada, ${ }^{1, *}$ S. Bustingorry, ${ }^{1}$ D. E. Pontello, ${ }^{1,2}$ M. Barturen, ${ }^{1,2,3}$ M. Eddrief,${ }^{3,4,5}$ M. Marangolo,,${ }^{3,4,5}$ and J. Milano ${ }^{1,2,3}$ \\ ${ }^{1}$ Consejo Nacional de Investigaciones Científicas y Técnicas, Centro Atómico Bariloche - CNEA, \\ (R8402AGP) San Carlos de Bariloche, Río Negro, Argentina \\ ${ }^{2}$ Instituto Balseiro, Universidad Nacional de Cuyo, Centro Atómico Bariloche, (R8402AGP) San Carlos de Bariloche, Argentina \\ ${ }^{3}$ LIFAN, Laboratorio Internacional Franco-Argentino en Nanociencias \\ ${ }^{4}$ Sorbonne Universités, UPMC Université Paris 06, UMR 7588, INSP, 4 place Jussieu, F-75252 Paris, France \\ ${ }^{5}$ CNRS, UMR 7588, Institut des Nanosciences de Paris, 4 place Jussieu, F-75005, Paris, France \\ (Received 23 March 2016; revised manuscript received 7 October 2016; published 29 November 2016)
}

\begin{abstract}
Magnetotransport properties of $\mathrm{Fe}_{0.8} \mathrm{Ga}_{0.2}$ films with stripe domains are studied. The anisotropic magnetoresistance dominates the low field behavior, which is extremely dependent on the magnetic domains configuration. The magnetoresistance measured at different temperatures displays qualitatively different behaviors depending on the measurement configuration. When the stripes are oriented along the electric current, the low-field magnetoresistance changes sign with temperature, while when the stripes are perpendicular to the electric current the magnetoresistance curves are nearly temperature independent. A simple model considering parallel (series) conduction along (across) the stripes, plus the temperature dependence of anisotropic magnetoresistance and domains configuration, accounts for these experimental results.
\end{abstract}

DOI: 10.1103/PhysRevB.94.184435

\section{INTRODUCTION}

$\mathrm{Fe}_{1-x} \mathrm{Ga}_{x}$ alloys have been widely studied in the past decade due to their relatively high magnetostriction coefficient, roughly 10 times larger than that for iron, at moderate magnetic fields [1]. In recent years, $\mathrm{Fe}_{1-x} \mathrm{Ga}_{x}$ magnetostrictive films have been studied in thin film form, deposited either by sputtering [2-4], molecular beam epitaxy (MBE) [5-7], electrodeposition [8], or specially designed techniques $[9,10]$. Interesting applications in devices based on $\mathrm{Fe}_{1-x} \mathrm{Ga}_{x}$ films have been proposed, like microwave filters [7] or magnetic field sensors [2,3]. The structure and magnetic properties of MBEgrown $\mathrm{Fe}_{1-x} \mathrm{Ga}_{x}$ films were thoroughly studied [5,6] and some remarkable differences compared to the bulk material were found. In particular, magnetic domains with stripe geometry were observed in films with thickness above a threshold value that depends on Ga concentration [11]. The stripe domains, observed in the remanent state, are aligned along the direction of the last visited in-plane homogeneous magnetic state. It costs a certain energy to deviate them due to the rotatable magnetic anisotropy that favors such orientation [12-16], making the stripe domains configuration quite stable. The origin of striped magnetic domains is ascribed to the presence of a perpendicular magnetic anisotropy [17] competing with dipolar interaction, and they are present in films of a wide variety of ferromagnetic materials [18].

As demonstrated in Ref. [3], the effect of a magnetic field on $\mathrm{Fe}_{1-x} \mathrm{Ga}_{x}$ films can be detected with a good sensitivity by measuring the electrical resistance of the device. This suggests that resistivity measurements could be an appealing way of sensing variations in the magnetic structure of these films, either induced directly by an applied magnetic field or indirectly by a mechanical deformation as a consequence of magnetoelastic coupling. For exploring this possibility, the electronic transport properties of thin films need to be characterized.

*Corresponding author: granadam@cab.cnea.gov.ar
Magnetotransport studies of films of different ferromagnetic metals presenting stripe domains can be found in the literature. There was a first attempt to describe the low-field increase in the magnetoresistance (MR) measured on Co films with stripe domains using models inspired in the giant MR (GMR) phenomenon observed in ferromagnetic/nonmagnetic multilayers [19,20]. However, subsequent works reported on a negative contribution to the resistivity due to domain walls in lithography patterned Fe films [21-23], inconsistent with a GMR-like behavior. Besides this negative domain wall resistance in $\mathrm{Fe}$, the main contribution to low-field magnetoresistance in $\mathrm{Fe}, \mathrm{Co}$, and other materials was found to be the anisotropy of the resistivity, either due to anisotropic MR (AMR) or Lorentz MR [24-26].

In this work we present a study on the magnetotransport properties of a $\mathrm{Fe}_{0.8} \mathrm{Ga}_{0.2}$ film with stripe domains. Magnetoresistance was measured as a function of temperature and varying the angle between electric current and magnetic field. The magnetotransport properties of the system are dominated by AMR and details of the magnetic domains structure, as observed previously in Fe and Co films with stripe domains [24]. A qualitatively different behavior, depending on the angle between the stripes and the electric current, is observed in the low-field magnetoresistance as a function of temperature. When the magnetic field is applied perpendicular to the current, the MR curves are roughly temperature independent. However, if the field is applied parallel to the current, the shape of MR curves changes dramatically with temperature, reversing the sign of the low-field MR. This behavior is explained as due to parallel or series conduction taking place along or across the stripes, respectively. The results are interpreted in the framework of a simplified model for the magnetic structure, in which closure domains play a crucial role.

\section{SAMPLE PREPARATION AND MAGNETIC CHARACTERIZATION}

Single crystalline $\mathrm{Fe}_{1-x} \mathrm{Ga}_{x}$ films were grown by molecular beam epitaxy on (001) GaAs substrates. A 20-nm ZnSe 
(a)
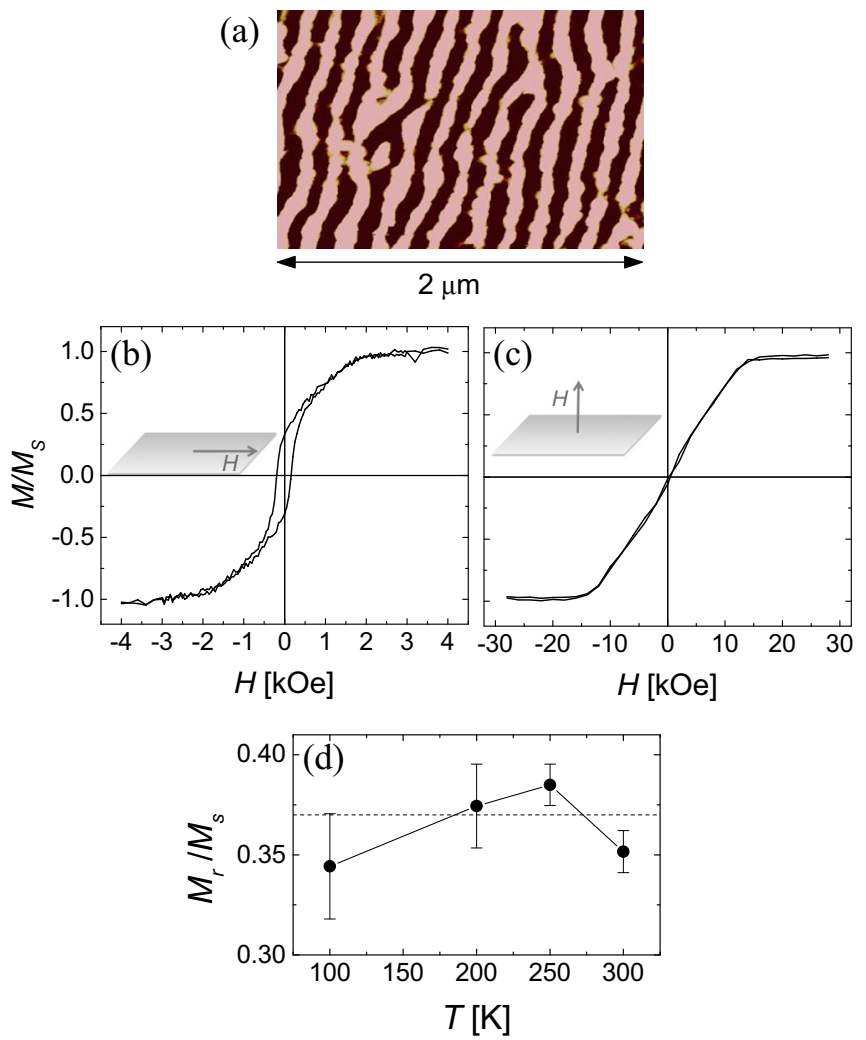

FIG. 1. (a) Magnetic force microscopy image of 72-nm-thick $\mathrm{Fe}_{0.8} \mathrm{Ga}_{0.2}$ film. The image was acquired at room temperature and at zero applied field; the stripes lie along the direction of the previously applied magnetic field. (b) In-plane and (c) out-of-plane magnetization loops measured at $300 \mathrm{~K}$ in the same sample. (d) Normalized remanent magnetization obtained from in-plane magnetization loops at different temperatures. The dashed line, $M_{r} / M_{s}=0.37$, represents the mean value of the data.

buffer layer was used to avoid interdiffusion between the $\mathrm{Fe}_{1-x} \mathrm{Ga}_{x}$ film and the substrate, and a 3-nm gold capping layer prevents the metallic film from oxidizing. Details on the fabrication procedure and structural characterization are given elsewhere [6]. In the present work we study a $\mathrm{Fe}_{0.8} \mathrm{Ga}_{0.2}$ film of $72 \mathrm{~nm}$ nominal thickness, which presents a stripe domains structure [11]. The stripes are formed when decreasing the magnetic field from saturation and they are oriented in the direction of the last in-plane saturating field. Figure 1(a) shows a typical magnetic force microscopy image of the sample at remanence. The stripes observed at room temperature have an average period of $160 \mathrm{~nm}$.

Magnetization loops measured at room temperature are presented in Figs. 1(b) and 1(c). The magnetization was normalized to the saturation value $M_{s}$. The in-plane saturation field is of $\sim 2 \mathrm{kOe}$, and the linear behavior characteristic of the stripes structure [11] is observed below such field [see Fig. 1(b)]. When the field is applied perpendicular to the sample, the saturation field is $\sim 13 \mathrm{kOe}$ [see Fig. 1(c)], the outof-plane direction being a hard axis of magnetization. Similar measurements were performed at different temperatures in the range between 100 and $300 \mathrm{~K}$. The in-plane saturation and coercive fields experience a slight increase with increasing temperature, giving evidence of the competition between dipolar interactions and magnetic anisotropies, as discussed in Ref. [27]. The remanent magnetization, presented in Fig. 1(d), does not show any temperature dependence, within the error bars. The measured $M_{r} / M_{s}$ data have an average value of 0.37 .

\section{MAGNETOTRANSPORT EXPERIMENTS}

The electrical resistance was measured in a standard fourprobe configuration, with collinear contacts along the [110] crystalline direction. The voltage contacts are separated by $1.5 \mathrm{~mm}$, so the effective size of the sample is much larger than the stripes period. The measurements were performed with a maximum DC electric current of $10 \mathrm{~mA}$, which gives a current density of $\sim 0.1 \mathrm{GA} / \mathrm{m}^{2}$, much lower than the current densities needed to induce domain wall displacement (e.g., some $\mathrm{TA} / \mathrm{m}^{2}$ for $\mathrm{Ni}_{81} \mathrm{Fe}_{19}$ [28]). Thus, we should not expect the electric current to affect the magnetic configuration at all. The field dependence of the resistivity was measured with the magnetic field applied at different angles from the electric current. Room temperature results are presented in Fig. 2.

\section{A. High-field magnetoresistance}

Above the direction-dependent saturation field, all the $\rho(H)$ curves follow a linear dependence with roughly the same slope. This behavior can be ascribed to electron-magnon scattering, as discussed in the literature for other ferromagnetic metals $[29,30]$. By extrapolating the high-field linear dependence to zero field, we obtain the resistivity that the sample would present at zero field if the magnetization was uniformly oriented along each direction [22], indicated as $\rho_{\|}, \rho_{\perp}$, and $\rho_{n}$ in Fig. 2. In the saturated regime, there is a resistivity difference between the curves due to AMR, i.e., the dependence of the resistivity on the angle between magnetization and electric current [31,32]. As shown in Fig. 2, the observed values of

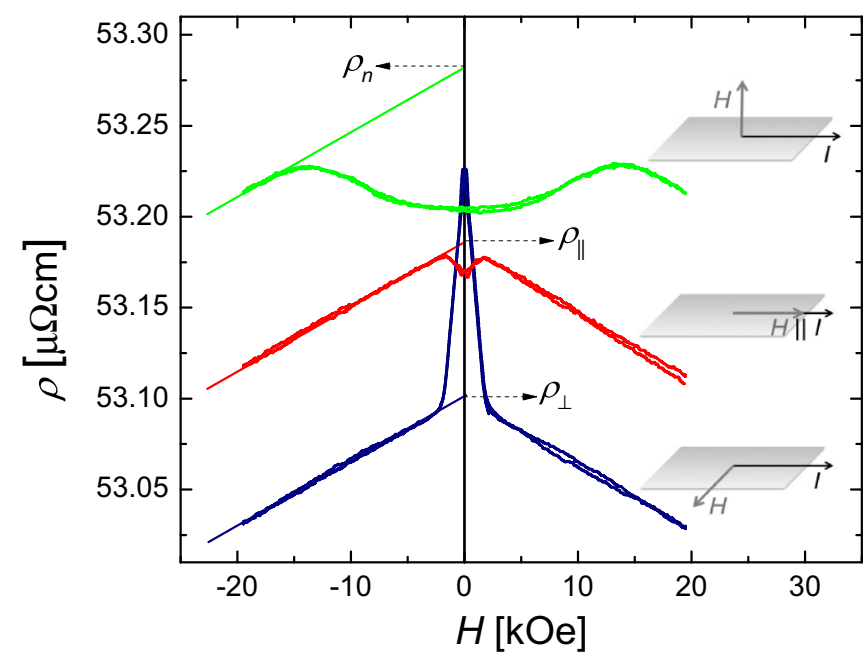

FIG. 2. Room temperature $\rho(H)$ curves measured with the magnetic field applied in the plane (blue and red) and normal to the plane of the sample (green). Schematics of current and field directions are shown at the right of each curve. The zero-field resistivities $\rho_{\perp}$, $\rho_{\|}$, and $\rho_{n}$ are extrapolated from the high-field linear behavior. 
zero-field extrapolated resistivities are such that

$$
\rho_{\perp}<\rho_{\|}<\rho_{n} .
$$

The nonequivalence between in-plane and out-of-plane perpendicular resistivities is known as geometrical size effect, and has been observed in films of Co and other materials [26].

\section{B. Low-field magnetoresistance}

Let us now focus on the behavior of the low-field resistivity for the in-plane measurements in Fig. 2. When the field is decreased after saturating the magnetization perpendicular to the current (blue curve), the resistivity increases as the field vanishes. In this field regime, the stripes are formed giving rise to an oscillating out-of-plane magnetization component $M_{z}$, whose maximum increases with vanishing field. This offplane canting of the magnetization will cause the resistivity to change toward $\rho_{n}$ which is higher than the resistivity for in-plane magnetization components, thus yielding an increase of the overall resistivity. However, the off-plane canting of the magnetization does not account for the curve measured with $\mathbf{H} \| I$, where the resistivity decreases for vanishing field. This decrease from the saturated $\rho_{\|}$resistivity would imply a fraction of material having in-plane magnetization component perpendicular to the current, since $\rho_{\perp}<\rho_{\|}$as discussed above. Indeed, the formation of stripes does not only consist of an off-plane canting of the magnetization but also of a fraction of material forming closure domains with an in-plane component of $\mathbf{M}$ perpendicular to the stripes [18]. A simulated domains structure for this material was presented by Tacchi et al. in Ref. [15]. One can see in Fig. 1(j) of Ref. [15] that the regions close to the top and bottom surfaces of the film have an in-plane component of the magnetization perpendicular to the stripes, and it would be this fraction of material that gives rise to the decreasing resistivity at vanishing field.

\section{Temperature dependence of the magnetoresistance}

In order to gain more insight into the relation between the measured resistivity and the inhomogeneous magnetization distribution, we performed MR measurements at different temperatures. The magnetoresistance curves, with

$$
\operatorname{MR}(H)[\%]=\frac{\rho(H)-\rho(0)}{\rho(0)} \times 100,
$$

are presented in Fig. 3. We present here MR instead of resistivity since the linear $\rho(T)$ dependence produces a $\rho$ variation larger than $0.1 \%$ in $1 \mathrm{~K}$, which in the studied temperature range would completely mask the $\rho(H)$ variation. As already observed in Fig. 2, the room temperature in-plane MR has opposite signs depending on whether the stripes are oriented perpendicular or parallel to the current [see Figs. 3(a) and 3(b)]. When the in-plane magnetic field is applied perpendicular to the electric current [Fig. 3(a)], the MR curves do not vary noticeably with temperature. Interestingly, when the in-plane magnetic field is parallel to the current [Fig. 3(b)], the shape of MR curves is highly temperature dependent, the low-field magnetoresistance changing from positive to negative with decreasing temperature. The MR curves measured with the magnetic field perpendicular to the
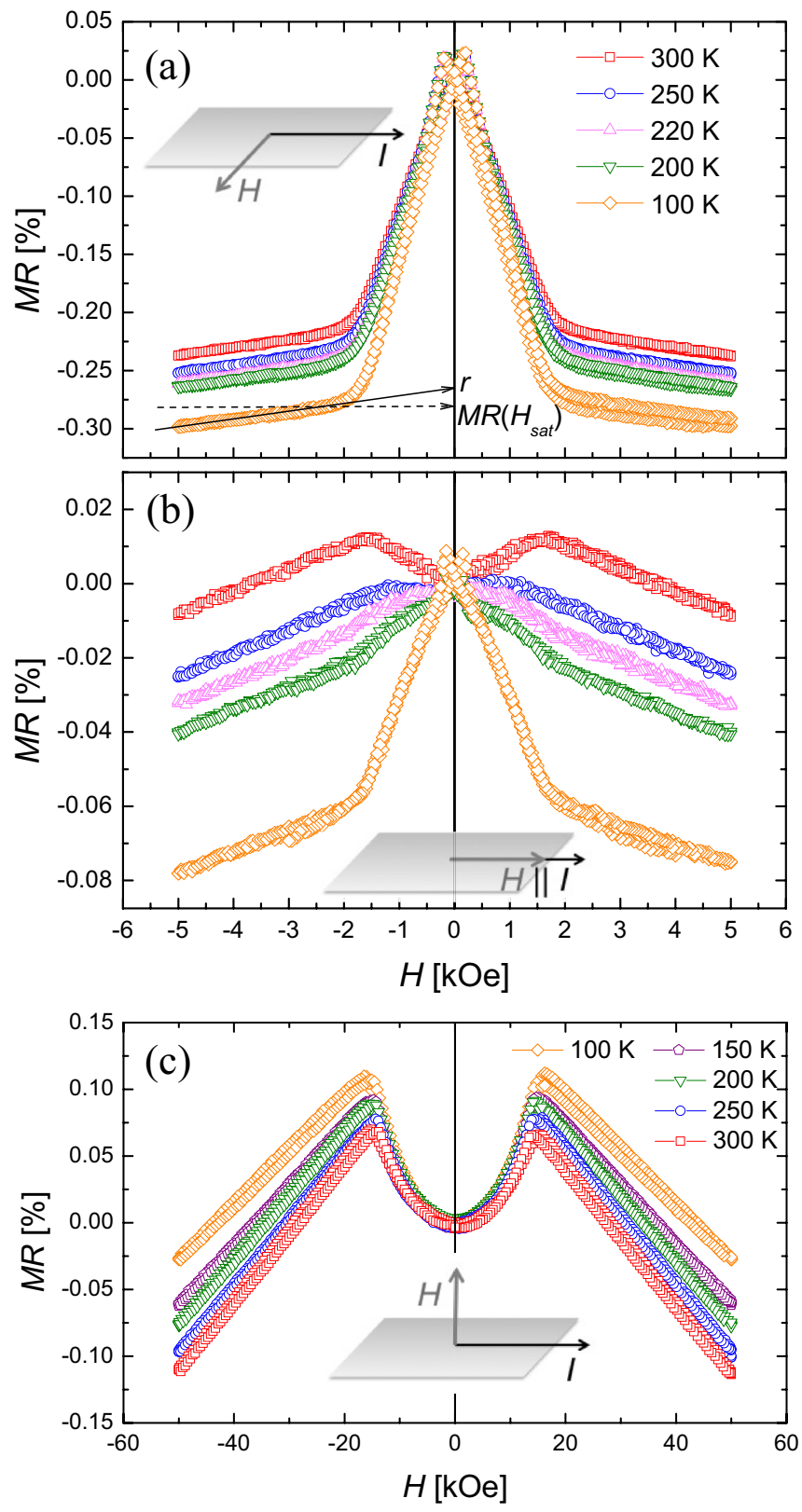

FIG. 3. Magnetoresistance measured at different temperatures between 100 and $300 \mathrm{~K}$ with the magnetic field applied (a) in the plane of the sample perpendicular to the current, (b) parallel to the current, and (c) perpendicular to the sample. In panel (a), the $r$ and $\operatorname{MR}\left(H_{\text {sat }}\right)$ parameters defined in the text are indicated for the $100-\mathrm{K}$ curve.

film are presented in Fig. 3(c). In this case, the shape of the curves is rather temperature independent.

For analyzing the temperature behavior of MR curves, the low-field and high-field slopes $s=d(\mathrm{MR}) / d H$ were computed and plotted in Fig. 4(a). The high-field slopes are very similar for all the configurations and their absolute values diminish slightly with decreasing temperature. This weak temperature dependence reflects the fact that electron-magnon scattering is almost unaltered in the temperature range between 100 and $300 \mathrm{~K}$. The magnon population is slightly reduced with decreasing temperature, and thus the effect of magnetic 


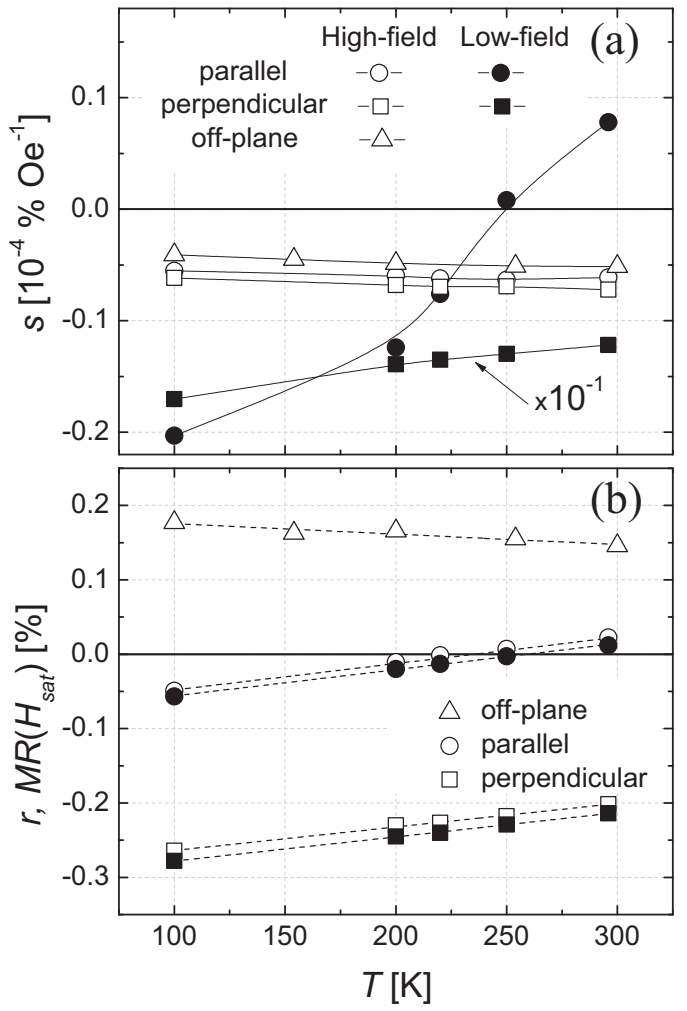

FIG. 4. (a) Slopes of $\operatorname{MR}(H)$ curves in Fig. 3 for the different magnetic-field configurations. The high- and low-field slopes correspond to the linear behavior observed above and below the saturation field, respectively. The low-field data in the perpendicular configuration are divided by 10. (b) Hollow symbols: zero-field intercept $r$ of the high-field linear behavior of $\operatorname{MR}(H)$ curves in Fig. 3; solid symbols: MR evaluated at saturation field, $\operatorname{MR}\left(H_{\text {sat }}\right)$ [see Fig. 3(a) for an example of $r$ and $\operatorname{MR}\left(H_{\text {sat }}\right)$ ]. The dashed lines in panel (b) are linear fits to data.

field is also reduced. On the contrary, the low-field slopes are more temperature dependent and dramatically affected by the magnetic field orientation. For the in-plane perpendicular configuration, the low-field slopes have large negative values, while in the parallel configuration the slopes are smaller and changing sign near $250 \mathrm{~K}$. These results will be discussed later in Sec. IV.

In the same way as we defined the resistivities extrapolated to zero field in Fig. 2, we can extrapolate the high-field linear dependence of MR curves in Fig. 3 to obtain the values $r_{\perp}, r_{\|}$, and $r_{n}$ for the perpendicular, parallel, and off-plane configurations, respectively. It is also useful to define $\operatorname{MR}\left(H_{\text {sat }}\right)$ as the MR evaluated at the saturation magnetic field, that is, the field delimiting the regimes with and without stripes recognized by a kink in MR curves. These parameters are plotted as a function of temperature in Fig. 4(b).

Finally, we measured the angular variation of the resistivity at different temperatures. The in-plane curves were measured with a 5-kOe applied field, and the out-of-plane curves with $80 \mathrm{kOe}$, in order to ensure that the magnetization follows the magnetic field direction in all cases. Figure 5 shows the angular dependence of the anisotropic magnetoresistance

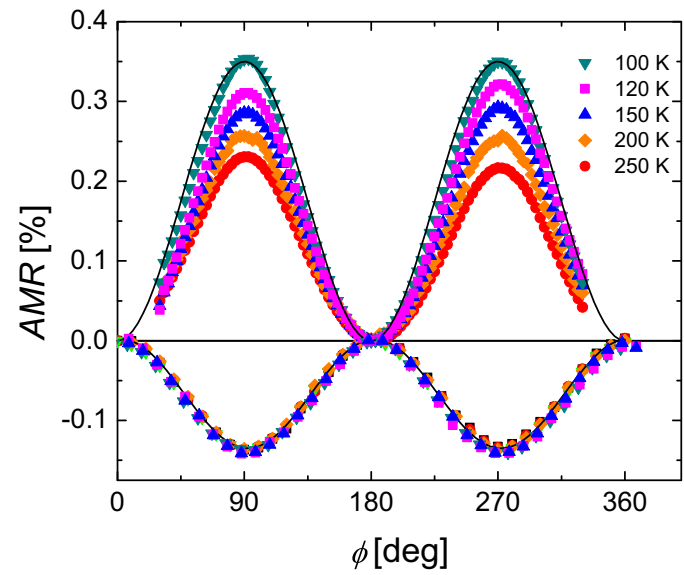

FIG. 5. AMR measured at different temperatures between 100 and $300 \mathrm{~K}$. The lines depict $\cos ^{2}(\phi)$ dependencies for in-plane $(\mathrm{AMR}<0)$ and out-of-plane (AMR $>0)$ angular variations. In both configurations, $\phi=0$ corresponds to the magnetic field parallel to the electric current direction. Continuous lines are $\cos ^{2}(\phi)$ fitting curves for $100-\mathrm{K}$ data.

AMR, defined as

$$
\operatorname{AMR}(\phi)[\%]=\frac{\rho(\phi)-\rho(0)}{\rho(0)} \times 100,
$$

where $\phi=0$ corresponds to the magnetic field parallel to the electric current direction, which lies along the [110] crystallographic axis in this work. The AMR is normalized here to the $\rho(\phi=0)$ value instead of $\rho_{a v}=\frac{1}{3} \rho_{\|}+\frac{2}{3} \rho_{\perp}$ as in the usual definition for polycrystalline samples [31], due to the difference between $\rho_{\perp}$ and $\rho_{n}$ found in the present films. All the measured curves can be fitted with a $\cos ^{2}(\phi)$ dependence [31] (lines in Fig. 5 represent two examples), which gives evidence that the magnetization is saturated along the field direction and then the $\rho(\phi)$ variation is due to conventional AMR. The in-plane AMR does not depend on temperature, indicating that $\rho_{\perp} / \rho_{\|}$is temperature independent as well. This is consistent with the fact that the difference between $\operatorname{MR}\left(H_{\mathrm{sat}}\right)_{\|}$and $\operatorname{MR}\left(H_{\text {sat }}\right)_{\perp}$ is roughly temperature independent [see Fig. 4(b)]. The out-of-plane AMR, on the other hand, shows increasing amplitude with decreasing temperature. This means that $\rho_{n} / \rho_{\|}$ changes with temperature while $\rho_{\perp} / \rho_{\|}$does not. The results indicate that Eq. (1) holds in all the studied temperature range.

Using Eq. (3) for the case $\phi=90^{\circ}$, one can compute $\rho_{n} / \rho_{\|}$ and $\rho_{\perp} / \rho_{\|}$. To do this, AMR is evaluated at $\phi=90^{\circ}$ for each curve in Fig. 5, and the resistivities are replaced by $\rho(0)=\rho_{\|}$and $\rho\left(90^{\circ}\right)=\rho_{n}$ or $\rho_{\perp}$, depending of the measurement configuration. Knowing the ratios $\rho_{n} / \rho_{\|}$and $\rho_{\perp} / \rho_{\|}$, one can also equate $\rho_{n} / \rho_{\perp}$. These resistivity ratios are plotted in Fig. 6.

In the preceding analysis, the assumption that the high-field slopes of $\operatorname{MR}(H)$ curves are equal in all directions is implicit, since the ratios in Fig. 6 are expressed in terms of the zerofield extrapolated resistivities, while the experiments were performed at different applied magnetic fields. Figure 4(a) shows that this assumption is a reasonable approximation. 


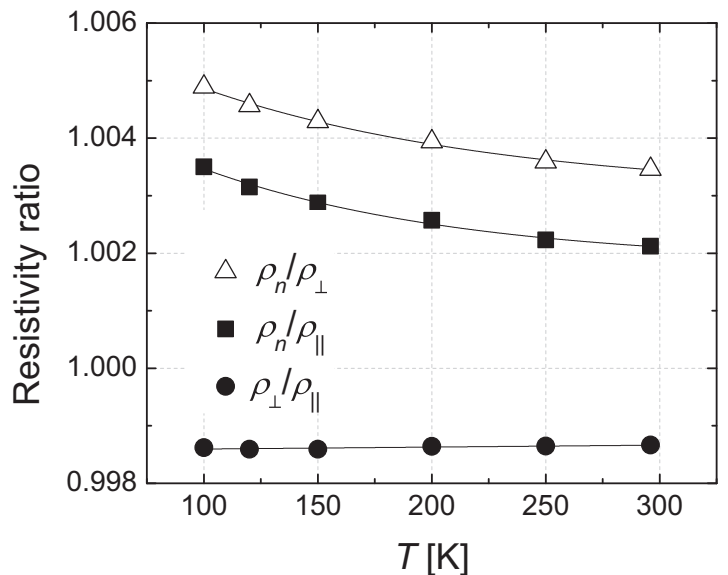

FIG. 6. Resistivity ratios $\rho_{\perp} / \rho_{\|}$and $\rho_{n} / \rho_{\|}$calculated from AMR curves in Fig. 5 as described in the text. The room-temperature $\rho_{n} / \rho_{\|}$ value was calculated directly from data in Fig. 2 . The $\rho_{n} / \rho_{\perp}$ ratio was computed from the other ones. The lines are fits to data, a linear fit in the case of $\rho_{\perp} / \rho_{\|}$and exponential fits for the other two cases.

The parameters plotted in Figs. 4(b) and 6 will be used in the following section for discussing the temperature dependence of the magnetic structure.

\section{DISCUSSION}

For the interpretation of magnetotransport results in Fig. 3, we will consider the local magnetization orientation and the dependence of the resistivity on the angle between $\mathbf{M}$ and electric current, i.e., the AMR. We propose here a simple analysis based on geometrical considerations that will allow for a qualitative discussion of the results.

Let us consider the simplified domains structure schematized in Fig. 7, similar to that used by Fin et al. [16]. In this model the magnetization is oriented along three directions, as represented by arrows in Figs. 7(a) and 7(b). The cores of the stripe domains have the magnetization normal to the plane of the film; this is a reasonable approximation based on a model considering the off-plane canting of the magnetization in the

(a)

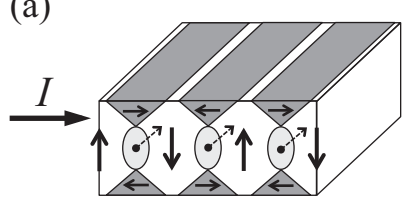

(b)

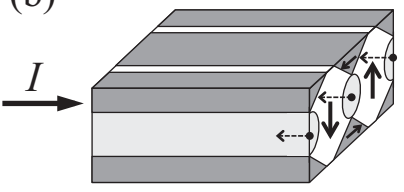

(c)

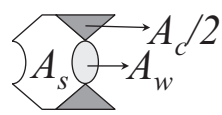

FIG. 7. Simplified magnetic structure consisting of stripe cores (white) with the magnetization in the direction normal to the film, closure domains (dark gray) with the magnetization in the plane of the film and perpendicular to the stripes, and Bloch walls (light gray) with the magnetization along the stripes direction. Once the stripes are formed, the electric current $I$ runs across (a) or along (b) the stripe domains. (c) The areas of stripe cores, $A_{s}$, closure domains, $A_{c}$, and Bloch walls, $A_{w}$, in one semiperiod are indicated. stripes [27] that allowed us to estimate the maximum canting angle $\theta=85^{\circ}$, quite close to the direction perpendicular to the film. The closure domains have the magnetization in the plane of the film and perpendicular to the stripes; there are also regions with the magnetization along the stripes, representing the Bloch walls. These approximations rely on micromagnetic calculations of the stripes structure $[15,16]$.

The magnetic domains in Fig. 7 are taken as the configuration in remanence $(H=0)$ and we will compare their resistance with the one at saturation. The magnetization will have only three possible directions: parallel to the current, perpendicular to the current in the plane of the sample, and perpendicular to the film plane. The domains with such magnetization orientations will have resistivities $\rho_{\|}, \rho_{\perp}$, and $\rho_{n}$, respectively (see Fig. 2). We now consider each domain as a resistor and approximate electronic transport taking place across the stripes [Fig. 7(a)] in an equivalent series circuit or along the stripes [Fig. 7(b)] in a parallel circuit. This system can be considered as ohmic since the mean free path is much smaller than the typical length scales of the magnetic structure. Using a resistivity of $47 \mu \Omega \mathrm{cm}$ measured for the present sample at $100 \mathrm{~K}$ and $r_{s} / a_{0}=2.12$ for pure iron from Ref. [33] (being $r_{s}$ the radius of the free electron sphere and $a_{0}$ the Bohr radius), we can estimate an upper limit for the electronic mean free path smaller than $9 \mathrm{~nm}$, while the periodicity of the stripes is of $160 \mathrm{~nm}$. In the discussion that follows, we will call $\rho\left(H_{\text {sat }}\right)=\rho^{\text {sat }}$ and $\rho(H=0)=\rho^{\text {rem }}$ for short, and use subindexes $\|$ and $\perp$ to indicate the measurement configuration, i.e., the direction of the applied magnetic field.

Let us analyze first the case with the in-plane magnetic field applied perpendicular to the current. In this case, the saturated state will have resistivity $\rho_{\perp}^{\text {sat }}=\rho_{\perp}$. In remanence, the stripes lie perpendicular to the current as depicted in Fig. 7(a), with domain walls of resistivity $\rho_{\perp}$ surrounded by regions of higher resistivity [see Eq. (1)]. Thus, in the perpendicular configuration the resistivity of the sample with stripes, $\rho_{\perp}^{\mathrm{rem}}$, will be higher than the resistivity of the homogeneously magnetized sample, $\rho_{\perp}^{\text {sat }}$. This is always the case independently of the relative areas in Fig. 7(c) and is in coincidence with the results in Fig. 3(a).

On the other hand, when the field is applied parallel to the current, the remanence configuration needs to be considered as parallel resistors [see Fig. 7(b)] and the net resistivity is calculated as

$$
\rho_{\|}^{\mathrm{rem}}=\left(\frac{a_{s}}{\rho_{n}}+\frac{a_{c}}{\rho_{\perp}}+\frac{a_{w}}{\rho_{\|}}\right)^{-1}
$$

with $a_{i}=A_{i} / A$ and $A=A_{s}+A_{c}+A_{w}$ [see Fig. 7(c) for the definition of the areas]. Using the relation

$$
a_{s}+a_{c}+a_{w}=1,
$$

Eq. (4) can be rewritten as follows:

$$
\rho_{\|}^{\mathrm{rem}}=\rho_{\|}\left[1+a_{s}\left(\frac{1}{\rho_{n} / \rho_{\|}}-1\right)+a_{c}\left(\frac{1}{\rho_{\perp} / \rho_{\|}}-1\right)\right]^{-1} .
$$

One can see that the remanence resistivity $\rho_{\|}^{\text {rem }}$ can be either lower or higher than the saturation resistivity $\rho_{\|}^{\text {sat }}=\rho_{\|}$, depending on the ratios between resistivities and the relative 

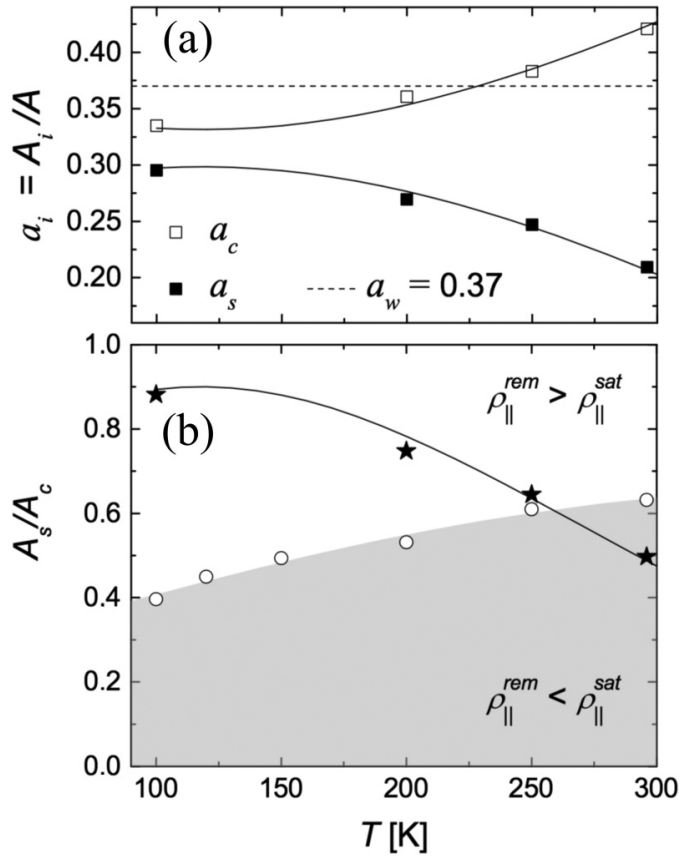

FIG. 8. (a) Temperature dependence of the relative areas of stripe cores $\left(a_{s}\right)$ and closure domains $\left(a_{c}\right)$ estimated from $\operatorname{MR}(H)$ curves as described in the text, by considering a constant value for $a_{w}$. (b) Volume ratio of stripe and closure domains, $A_{s} / A_{c}$. The white and gray regions correspond to negative and positive low-field MR, respectively. The boundary was determined by imposing the condition $\rho_{\|}^{\text {rem }}=\rho_{\|}^{\text {sat }}=\rho_{\|}$to Eq. (6) and using resistivity ratios obtained from $\operatorname{AMR}(\phi)$ measurements; the hollow circles were obtained using discrete data in Fig. 6 to perform this calculation and the continuous boundary was computed using the fitted curves to those data. $A_{s} / A_{c}$ values calculated from relative areas in panel (a) are also plotted (black stars).

areas $a_{i}$. The $A_{s} / A_{c}$ ratio defining the boundary between such regimes can be estimated as a function of temperature using Eq. (6) under the condition $\rho_{\|}^{\text {rem }}=\rho_{\|}^{\text {sat }}$ and using the resistivity ratios in Fig. 6. The result is presented in Fig. 8(b); the hollow dots were calculated using the discrete data in Fig. 6 and the continuous boundary between $\rho_{\|}^{\text {rem }}>\rho_{\|}^{\text {sat }}$ (white) and $\rho_{\|}^{\text {rem }}<$ $\rho_{\|}^{\text {sat }}$ (gray) regions was calculated using the fitted curves for those data. So far, we have used $\operatorname{AMR}(\phi)$ data. In what follows, we will estimate the actual values of $A_{s} / A_{c}$ by analyzing $\operatorname{MR}(H)$ results. Equation (2) evaluated at the saturation field, $\operatorname{MR}\left(H_{\text {sat }}\right)=\left(\rho^{\mathrm{sat}}-\rho^{\mathrm{rem}}\right) / \rho^{\mathrm{rem}} \times 100$, can be rewritten as

$$
\rho^{\text {sat }}=\left[1+\operatorname{MR}\left(H_{\text {sat }}\right) / 100\right] \times \rho^{\mathrm{rem}} .
$$

Specializing this equation for the parallel configuration, we can use $\rho_{\|}^{\text {sat }}=\rho_{\|}$and $\rho_{\|}^{\text {rem }}$ given by Eq. (4). Then, using Eq. (5) we can equate $a_{s}$ and $a_{c}$ as a function of $a_{w}$, the resistivity ratios and $\operatorname{MR}\left(H_{\text {sat }}\right)$ in Fig. 4(b). We now use the result in Fig. 1(d) to estimate $a_{w}$ : within the present model, Fig. 7, only Bloch walls give a finite contribution to the in-plane remanent magnetization, thus suggesting $a_{w}=M_{r} / M_{s}$. As discussed before, Fig. 1(d) does not show a clear temperature dependence for $M_{r} / M_{s}$; thus we will consider the average value $a_{w}=0.37$ for all temperatures to obtain $a_{s}$ and $a_{c}$. The relative areas are plotted as a function of temperature in Fig. 8(a). At $100 \mathrm{~K}$ the three areas are comparable, and as the temperature increases, the closure domains grow at the expense of stripe cores, while the volume of Bloch walls is kept constant. The $A_{s} / A_{c}$ values obtained from the above defined $a_{s}$ and $a_{c}$ are presented as black stars in Fig. 8(b). The $A_{s} / A_{c}$ ratio is found to vary with temperature in a way consistent with the sign change of low-field MR. This implies that relative areas $A_{s}, A_{c}$, and $A_{w}$ change with temperature accompanying the change of AMR. The variation of the domains structure with temperature might be a consequence of the temperature dependence of magnetic anisotropies studied in Ref. [27].

As deduced from the preceding discussion, the variation with temperature of the MR $(H)$ curves in Fig. 3(b) would be due to a combination of the temperature dependence of the resistivity ratios (or in other words, of the AMR) and of the domains structure. However, we shall keep in mind that the $a_{s}, a_{c}$, and $a_{w}$ values were computed within the oversimplified magnetic structure in Fig. 7, which actually represents effective areas proportional to the three orthogonal magnetization components. In order to obtain quantitative information, a more elaborate model for the magnetic structure should be used, like micromagnetic calculations $[15,16]$ or a model such as the one used by Murayama [34], considering magnetization elements with arbitrary directions. Nevertheless, the proposed model allowed us to explain qualitatively the main result: In the in-plane perpendicular configuration the resistivity in remanence is always higher than in saturation, while in the parallel configuration the resistivity in remanence can be either higher or lower than in saturation, depending on the ratios of the relative areas of the stripe cores, closure domains, and Bloch walls.

\section{SUMMARY AND CONCLUSION}

In summary, we have measured magnetoresistance with the magnetic field applied parallel and perpendicular to the electric current at different temperatures. It is noteworthy that the room-temperature measurements of the in-plane MR with the stripes oriented parallel or perpendicular to the electric current present opposite sign in the low-field region. While in the perpendicular cases the MR curves do not change much with temperature, in the parallel case the shape of the curves is very much temperature dependent, with a sign reversal of the low-field MR at $\sim 250 \mathrm{~K}$. The in-plane curves in the presence of stripe domains were described in terms of parallel or series conduction, considering an equivalent circuit where the different resistors represent the core of stripe domains, closure domains, and Bloch walls. Associating to each magnetization orientation different resistivities due to AMR, and comparing for each configuration the remanence and saturation MR at different temperatures, we deduced that the closure domains grow at the expense of stripe cores with increasing temperature.

Magnetotransport experiments have proved to be very sensitive to the magnetic configuration of the sample. Furthermore, they can efficiently detect changes in the magnetization components perpendicular to the field direction that cannot be studied by global magnetometry techniques. The magnetic moments of stripe cores and closure domains alternate in sign (see Fig. 7), yielding a vanishing net magnetic moment in 
the directions perpendicular to the magnetic field. However, although the magnetization of stripe cores and closure domains is compensated, the resistivity of all domains contribute to the total electrical resistance, allowing for their detection.

We have provided strong evidence that a temperature variation may affect the domain configuration as well as the AMR of the studied material. However, some limitations arise if one wants to extract quantitative information. The model used for the discussion (Fig. 7) is oversimplified, considering magnetization with only three allowed directions and neglecting the canting process during the formation of the stripes. To overcome these limitations, accurate simulations of the magnetic structure at different temperatures need to be performed.

Finally, Fig. 3 suggests that if $\mathrm{Fe}_{1-x} \mathrm{Ga}_{x}$ films with stripe domains are to be used as magnetic field sensors by measuring their resistivity, the perpendicular in-plane configuration should be used, since the MR curves present a negligible temperature dependence in that geometry. On the contrary, using the parallel configuration, temperaturesensitive detection can be performed.

\section{ACKNOWLEDGMENTS}

The authors acknowledge M. Vásquez Mansilla and A. Butera for fruitful discussions. This work was supported with funding from LIFAN; Agencia Nacional de Promoción Científica y Tecnológica (Argentina), PICT 2010-0773 and PICT 2013-0014; Universidad Nacional de Cuyo (Argentina), 06/C446; CONICET (Argentina), PIP 11220120100250CO; Agence Nationale de la Recherche (France), 13-JS04-0001-01 "SPINSAW".
[1] A. E. Clark, K. B. Hathaway, M. Wun-Fogle, J. B. Restorff, T. A. Lograsso, V. M. Keppens, G. Petculescu, and R. A. Taylor, J. Appl. Phys. 93, 8621 (2003).

[2] P. Zhao, Z. Zhao, D. Hunter, R. Suchoski, C. Gao, S. Mathews, M. Wuttig, and I. Takeuchi, Appl. Phys. Lett. 94, 243507 (2009).

[3] H. Bhaskaran, M. Li, D. Garcia-Sanchez, P. Zhao, I. Takeuchi, and H. X. Tang, Appl. Phys. Lett. 98, 013502 (2011).

[4] S. U. Jen and C. C. Liu, J. Appl. Phys. 115, 013909 (2014).

[5] A. McClure, H. Li, and Y. U. Idzerda, J. Appl. Phys. 107, 09A924 (2010).

[6] M. Eddrief, Y. Zheng, S. Hidki, B. Rache Salles, J. Milano, V. H. Etgens, and M. Marangolo, Phys. Rev. B 84, 161410(R) (2011).

[7] B. K. Kuanr, R. E. Camley, Z. Celinski, A. McClure, and Y. Idzerda, J. Appl. Phys. 115, 17C112 (2014).

[8] E. C. Estrine, W. P. Robbins, M. M. Maqableh, and B. J. H. Stadler, J. Appl. Phys. 113, 17A937 (2013).

[9] N. A. Morley, A. Javed, and M. R. J. Gibbs, J. Appl. Phys. 105, 07A912 (2009).

[10] A. Javed, N. A. Morley, and M. R. J. Gibbs, J. Appl. Phys. 107, 09A944 (2010).

[11] M. Barturen, B. Rache Salles, P. Schio, J. Milano, A. Butera, S. Bustingorry, C. Ramos, A. J. A. de Oliveira, M. Eddrief, E. Lacaze, F. Gendron, V. H. Etgens, and M. Marangolo, Appl. Phys. Lett. 101, 092404 (2012).

[12] S. S. Lehrer, J. Appl. Phys. 34, 1207 (1963).

[13] L. M. Alvarez-Prado, G. T. Pérez, R. Morales, F. H. Salas, and J. M. Alameda, Phys. Rev. B 56, 3306 (1997).

[14] M. Barturen, M. Sacchi, M. Eddrief, J. Milano, S. Bustingorry, H. Popescu, N. Jaouen, F. Sirotti, and M. Marangolo, E. Phys. J. B 86, 191 (2013).

[15] S. Tacchi, S. Fin, G. Carlotti, G. Gubbiotti, M. Madami, M. Barturen, M. Marangolo, M. Eddrief, D. Bisero, A. Rettori, and M. G. Pini, Phys. Rev. B 89, 024411 (2014).

[16] S. Fin, R. Tomasello, D. Bisero, M. Marangolo, M. Sacchi, H. Popescu, M. Eddrief, C. Hepburn, G. Finocchio, M. Carpentieri, A. Rettori, M. G. Pini, and S. Tacchi, Phys. Rev. B 92, 224411 (2015).
[17] M. Barturen, J. Milano, M. Vásquez-Mansilla, C. Helman, M. A. Barral, A. M. Llois, M. Eddrief, and M. Marangolo, Phys. Rev. B 92, 054418 (2015).

[18] A. Hubert and R. Schäfer, in Magnetic Domains: The Analysis of Magnetic Microstructures (Springer-Verlag, Berlin, Germany, 1998).

[19] J. F. Gregg, W. Allen, K. Ounadjela, M. Viret, M. Hehn, S. M. Thompson, and J. M. D. Coey, Phys. Rev. Lett. 77, 1580 (1996).

[20] P. M. Levy and S. Zhang, Phys. Rev. Lett. 79, 5110 (1997).

[21] U. Ruediger, J. Yu, S. Zhang, A. D. Kent, and S. S. P. Parkin, Phys. Rev. Lett. 80, 5639 (1998).

[22] A. D. Kent, U. Ruediger, J. Yu, S. Zhang, P. M. Levy, Y. Zhong, and S. S. P. Parkin, IEEE Trans. Mag. 34, 900 (1998).

[23] U. Rüdiger, J. Yu, A. D. Kent, and S. S. P. Parkin, Appl. Phys. Lett. 73, 1298 (1998).

[24] A. D. Kent, U. Rüdiger, J. Yu, L. Thomas, and S. S. P. Parkin, J. Appl. Phys. 85, 5243 (1999).

[25] A. D. Kent, J. Yu, U. Rüdiger, and S. S. P. Parkin, J. Phys.: Condens. Matter 13, R461 (2001).

[26] W. Gil, D. Görlitz, M. Horisberger, and J. Kötzler, Phys. Rev. B 72, 134401 (2005).

[27] M. Di Pietro Martínez, J. Milano, M. Eddrief, M. Marangolo, and S. Bustingorry, J. Phys.: Condens. Matter 28, 136001 (2016).

[28] J. Torrejon, G. Malinowski, M. Pelloux, R. Weil, A. Thiaville, J. Curiale, D. Lacour, F. Montaigne, and M. Hehn, Phys. Rev. Lett. 109, 106601 (2012).

[29] A. P. Mihai, J. P. Attané, A. Marty, P. Warin, and Y. Samson, Phys. Rev. B 77, 060401(R) (2008).

[30] B. Raquet, M. Viret, E. Sondergard, O. Cespedes, and R. Mamy, Phys. Rev. B 66, 024433 (2002).

[31] T. R. McGuire and R. I. Potter, IEEE Trans. Magn. 11, 1018 (1975).

[32] For a review on AMR effect in ferromagnetic metals, see I. A. Campbell and A. Fert, in Ferromagnetic Materials, edited by E. P. Wohlfarth (North Holland, Amsterdam, 1982), Vol. 3.

[33] N. W. Ashcroft and N. D. Mermin, Solid State Physics (Harcourt College Publishers, Fort Worth, TX, 1976).

[34] Y. Murayama, J. Phys. Soc. Jpn. 21, 2253 (1966). 